\title{
In vitro study of low intensity ultrasound combined with different doses of PDT: Effects on C6 glioma cells
}

\author{
JIAN-HUA LI ${ }^{1}$, ZHI-QIANG CHEN ${ }^{1}$, ZHENG HUANG ${ }^{2}$, QI ZHAN ${ }^{1}$, \\ FU-BIN REN ${ }^{1}$, JING-YE LIU ${ }^{1}$, WU YUE ${ }^{1}$ and ZHI WANG ${ }^{1}$ \\ ${ }^{1}$ Department of Neurosurgery, The Fourth College Hospital of Harbin Medical University, Harbin, P.R. China; \\ ${ }^{2}$ Department of Radiation Oncology, Colorado University of Health Sciences Center, Denver, CO, USA
}

Received July 26, 2012; Accepted October 16, 2012

DOI: $10.3892 / 01.2012 .1060$

\begin{abstract}
The aim of this study was to study the effects of killing C6 glioma cells induced by hematoporphyrin monomethyl ether (HMME)-mediated sonodynamic therapy combined with photodynamic therapy (SPDT). In the SPDT group, the cells were treated with sonication at an intensity of $0.5 \mathrm{~W} / \mathrm{cm}^{2}$ and a frequency of $1 \mathrm{MHz}$, followed by different doses of light irradiation. The growth inhibition rate following treatment was determined by MTT assay. The apoptotic rate was examined by a flow cytometry. Cleavage of caspase 3, 8 and 9 was investigated by immunoblotting. Reactive oxygen species (ROS) were measured by a fluorescence microplate reader. The effect of SPDT on the glioma cells was also studied in the absence or presence of various ROS scavengers. The growth inhibition rate of C6 glioma cells treated with SPDT was significantly higher compared with sonodynamic therapy (SDT) or photodynamic therapy (PDT) alone at light doses $<200 \mathrm{~J} / \mathrm{cm}^{2}$. The growth inhibition rate of $\mathrm{C} 6$ glioma cells treated with SPDT did not rise significantly when the light dose increased to $>120 \mathrm{~J} / \mathrm{cm}^{2}$. The apoptosis rate was the highest in the SPDT group, when the light dose was at $80 \mathrm{~J} / \mathrm{cm}^{2}$. A greater amount of ROS were generated in the SPDT group than in the groups treated with SDT or PDT alone. The addition of $\mathrm{NaN}_{3}$ or mannitol resulted in a decrease in the growth inhibition rate with SPDT. In conclusion, our data indicate that SPDT powerfully kills C6 glioma cells in vitro through the synergistic effects of SDT and PDT. The pathway of PDT inducing $\mathrm{C} 6$ glioma cell apoptosis includes both the mitochon-
\end{abstract}

Correspondence to: Professor Zhi Wang or Professor Wu Yue, Department of Neurosurgery, The Fourth College Hospital of Harbin Medical University, Harbin, P.R. China

E-mail: wangzhi992001@yahoo.com.cn

E-mail: yuewu@vip.163.com

Key words: sonodynamic and photodynamic therapy, hematoporphyrin monomethyl ether, apoptosis, reactive oxygen species, C6 glioma cells drial and death receptor pathways. Furthermore, ROS may play an important role in SPDT.

\section{Introduction}

Photodynamic therapy (PDT) is widely used in the therapy of gliomas at a biological level and in the clinic. PDT-mediated generation of molecular oxygen or reactive oxygen species (ROS) is assumed to be the main mechanism of cell death (1), and plays an important role in killing glioma cells. However, PDT has certain disadvantages; for example, its initial killing effect is not satisfactory. The effective depth of PDT is limited, as lasers are unable to penetrate and reach deep tissues to activate the photosensitizer.

Ultrasound has an appropriate tissue attenuation coefficient for penetrating and reaching deep-seated tissues while maintaining the ability to focus energy onto a small volume. This unique advantage makes it more useful for noninvasive treatment of deep-seated tumors when compared with electromagnetic modalities, such as laser beams (2-4). These techniques may be applied to the treatment of numerous types of cancer. However, a study has reported that the delayed killing effect is unsatisfactory in sonodynamic therapy (SDT), and advise that an alternative therapeutic method should be combined with PDT (5).

It is of note that certain sensitizers can be activated photochemically as well as sonochemically (6-8), such as porphyrin sonosensitizers $(3,4)$, as well as others (9-11). PDT combined with SDT (SPDT) is a new cancer therapy. The theory of the therapy is that by activating these congenerous sensitizers with light and sound, more cytotoxicity is generated to kill tumor cells. A number of scholars have reported the effects of killing tumor cells by SPDT; for example, Kessel et al (12) demonstrated that photodamage following exposure to ultrasound decreased the viability of murine leukemia L1210 cells which had survived ultrasonic treatment. Jin et al (13) studied SPDT for improving tumoricidal effects in a transplantable mouse squamous cell carcinoma (SCC) model, and showed that combination therapy induced tumor necrosis 2-3 times as deep as with either of the single modalities, concluding that SPDT may be useful in the treatment of non-superficial or 
nodular tumors. Kolarova et al (14) demonstrated that more ROS were generated using SPDT than using a monotherapy of PDT or SDT.

Nonetheless, research in this domain is rare, and to date, the effects of SPDT and the biological mechanisms by which it kills different tumor cell lines are undefined. Therefore, in the present study, we investigated the mechanisms by which PDT combined with SDT activates hematoporphyrin monomethyl ether (HMME) to kill C6 rat gliomas cells. We also studied the interactions between the two modalities.

\section{Materials and methods}

Cell cultivation. Rat glioma C6 cells were obtained from the Neurosurgery Institution of Harbin Medical University, China. C6 cells were maintained as monolayers in Roswell Park Memorial Institute (RPMI)-1640 medium (Hyclone Lab, Logan, UT, USA) at $37^{\circ} \mathrm{C}$ with $5 \% \mathrm{CO}_{2}$ in a humidified incubator (Nuaire, Plymouth, MN, USA). Cells in the exponential phase of growth were used for all the following experiments. The study was approved by the Ethics Committee of Haerbin Medical University, Harbin, China.

Sonodynamic and photodynamic treatment. All operations were carried out at $37^{\circ} \mathrm{C}$. Exponentially growing cells were collected by centrifugation, resuspended $\left(5 \times 10^{6}\right.$ cells $\left./ \mathrm{ml}\right)$ in serum-free RPMI-1640 medium and incubated with HMME for $4 \mathrm{~h}$. In the experiments, a multifunction physical therapy ultrasound device (Tianshi Technologies Ltd. Co, Beijing, China) was used to generate ultrasound at $1 \mathrm{MHz}$ in cells in the presence of $10 \mu \mathrm{g} / \mathrm{ml}$ HMME. Ultrasonic intensities $\left(0.5 \mathrm{~W} / \mathrm{cm}^{2}\right)$ were measured by a stainless steel ball radiometer (diameter, $0.32 \mathrm{~cm}$ ) (15). Further details are provided in a previous study by Li et al (16). Following this, cells were irradiated with a certain dose of light. For the irradiation, the wavelength was limited to $630 \mathrm{~nm}$ by an interference filter. The light was set to $100 \mathrm{~mW} / \mathrm{cm}^{2}$ for subsequent irradiation up to different doses of light using a semiconductor diode laser (Diomed 630, Andover, MA, USA).

The samples were divided into different groups. In the control group, the cells were not treated with HMME, nor with ultrasound or light irradiation. In the HMME group, the cells were treated with HMME but not with ultrasound or light irradiation. In the ultrasound and light irradiation groups, the cells were treated with ultrasound or light irradiation alone. In the SDT group, the cells were treated with both HMME $(10 \mu \mathrm{g} / \mathrm{ml})$ and ultrasound. In the PDT group, the cells were treated with both HMME $(10 \mu \mathrm{g} / \mathrm{ml})$ and light irradiation. In the SPDT group, the cells were treated with HMME $(10 \mu \mathrm{g} / \mathrm{ml})$ and sonication at an intensity of $0.5 \mathrm{~W} / \mathrm{cm}^{2}$, followed by exposure of $90 \mathrm{sec}$; subsequently, cells were irradiated with different doses of laser light.

Cell survival assay. After the treatment cells were washed, re-suspended in RPMI-1640 medium and subjected to MTT [3-(4,5-dimethylthiazol-2-yl)-2,5-diphenyltetrazolium bromide] assay. MTT $(20 \mu 1 ; 5 \mathrm{mg} / \mathrm{ml})$ was added. Four hours later, $100 \mu \mathrm{l}$ DMSO was added to each well to dissolve the resulting formazan crystals. Absorbance was read at $490 \mathrm{~nm}$ using an enzyme-linked immunosorbent assay reader (SpectraMax; Molecular Devices, Sunnyvale, CA, USA). Survival rate was calculated as: Inhibition rate $(\%)=$ (1 - $\left.\mathrm{OD}_{\text {treatment group }} / \mathrm{OD}_{\text {control group }}\right) \times 100$, where OD indicates optical density.

Examination of apoptosis or necrosis. Following treatment, cells were re-incubated for up to $4 \mathrm{~h}$ in the dark and washed twice with phosphate-buffered saline (PBS). After adjusting the cell density to $1 \times 10^{6}$ cells $/ \mathrm{ml}, 100 \mu \mathrm{l}$ cell suspension was transferred to a culture tube and mixed with $5 \mu \mathrm{l}$ Annexin V-FITC (fluorescein isothiocyanate; BD, Franklin Lakes, NJ, USA) and $5 \mu \mathrm{l}$ propidium iodide (PI; BD). The mixture was gently vortexed and incubated at room temperature $\left(25^{\circ} \mathrm{C}\right)$ in the dark for $15 \mathrm{~min}$. After adding $400 \mu \mathrm{l}$ binding buffer, the apoptotic rate was analyzed using a flow cytometer (BD).

Western blot analysis of caspase 3, 8 and 9 activation. Following treatment, cells were re-incubated for up to $4 \mathrm{~h}$ in the dark. To examine caspase 3,8 and 9 activation, cells of different groups were separately washed, collected and homogenized in a lysis buffer $(10 \mathrm{mM}$ Tris- $\mathrm{HCl}, \mathrm{pH} 8$, $0.32 \mathrm{mM}$ sucrose, $5 \mathrm{mM}$ EDTA, $2 \mathrm{mM}$ DTT, $1 \mathrm{mM}$ phenylmethyl sulfonylfluoride and $1 \%$ Triton X-100) and then centrifuged. Proteins in different groups were separately electrophoresed on SDS polyacrylamide gel (12\%), the gel-separated proteins were transferred to nitropure nitrocellulose membranes (Santa Cruz Biotechnology, Inc., Santa Cruz, CA, USA), and the membranes were probed overnight at $4^{\circ} \mathrm{C}$ with primary antibodies. Each of the targeted proteins was immunostained by distinct antibodies. The antibodies presented were: anti-actin, anti-cleavage caspase 3, 8 and 9 (Santa Cruz Biotechnology, Inc.). After probing, the membranes were washed three times and then incubated for $1 \mathrm{~h}$ at room temperature with the respective alkaline phosphatase- conjugated secondary antibodies (Sigma, St. Louis, MO, USA) before being visualized using a chemiluminescence detection kit (Sigma).

Measurement of ROS generation. 2',7'-Dichlorofluorescein diacetate (DCFH-DA; Beyotime Institute of Biotechnology, Shanghai, China) was used to detect SPDT-mediated ROS production. DCFH-DA was added to the cell suspension at a final concentration of $10 \mu \mathrm{mol} / 1$ and incubated at $37^{\circ} \mathrm{C}$ in the dark for $30 \mathrm{~min}$. Fluorescent dichlorofluorescein generated from the oxidation of DCFH-DA was measured by a fluorescence microplate reader (FLx800; BioTek, Winooski, VT, USA).

Cell survival assay following addition of different ROS scavengers. To confirm the involvement of ROS in SPDT, the above procedures (SDT) were repeated in the presence of either $100 \mu \mathrm{g} / \mathrm{ml}$ final concentration of sodium azide $\left(\mathrm{NaN}_{3}\right)$ or $100 \mu \mathrm{g} / \mathrm{ml}$ final concentration of mannitol $\left(\mathrm{C}_{6} \mathrm{H}_{14} \mathrm{O}_{6}\right)$. Subsequently, cells were subjected to an MTT assay.

Statistical analysis. Statistical evaluation was performed with a t-test using analytical software tools from SPSS. Data are presented as the mean values \pm standard error of the mean (SE). $\mathrm{P}<0.05$ was considered to indicate a statistically significant result. 


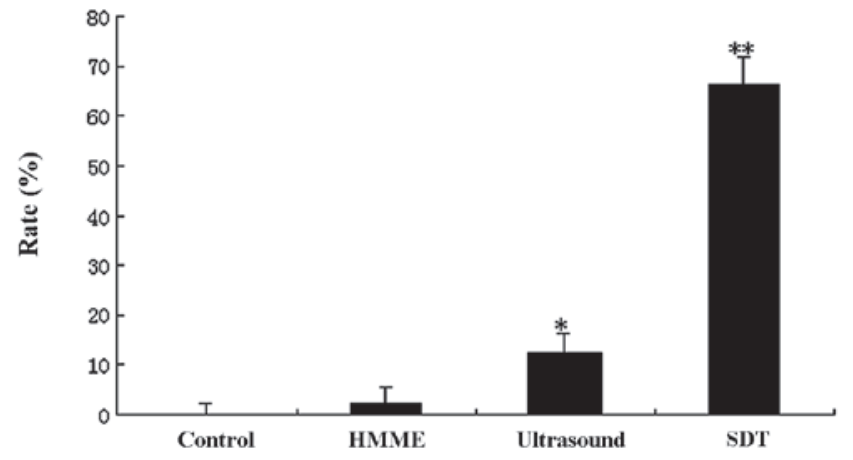

Figure 1. Growth inhibition rate of rat C6 glioma cells measured by MTT assay following sonodynamic therapy (SDT) treatment. Data represent mean $\pm \mathrm{SE}(\mathrm{n}=6) .{ }^{* *} \mathrm{P}<0.01$ vs. control, ${ }^{*} \mathrm{P}<0.05$ vs. control. HMME, hematoporphyrin monomethyl ether.

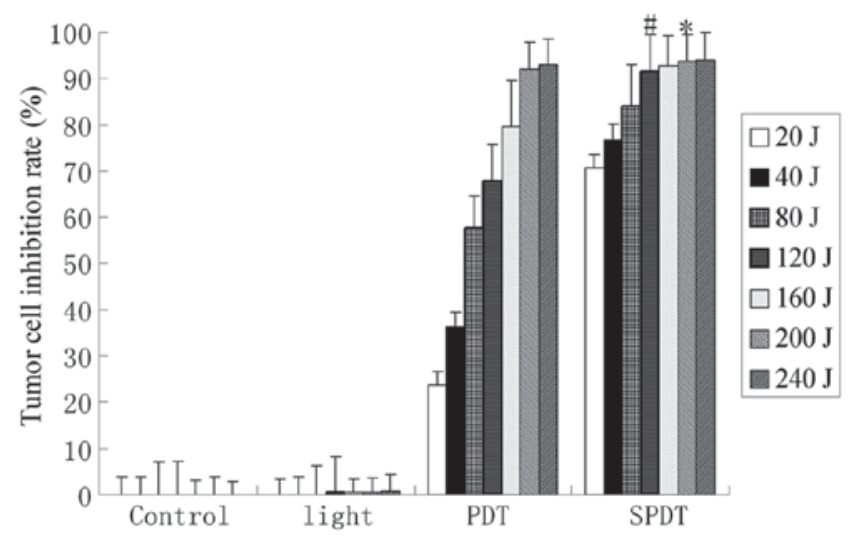

Figure 2. Growth inhibition rate of rat $\mathrm{C} 6$ glioma cells in different groups were measured by MTT assay. Data represents mean $\pm \mathrm{SE}(\mathrm{n}=6)$. ${ }^{*} \mathrm{P}<0.05$, SPDT vs. PDT (light irradiation dose $<200 \mathrm{~J} / \mathrm{cm}^{2}$ ). ${ }^{.} \mathrm{P}<0.05$, SPDT growth inhibition rate (light irradiation dose, $120 \mathrm{~J} / \mathrm{cm}^{2}$ ) vs. other groups of SPDT (light irradiation dose $<120 \mathrm{~J} / \mathrm{cm}^{2}$ ). SDT, sonodynamic therapy; PDT, photodynamic therapy.

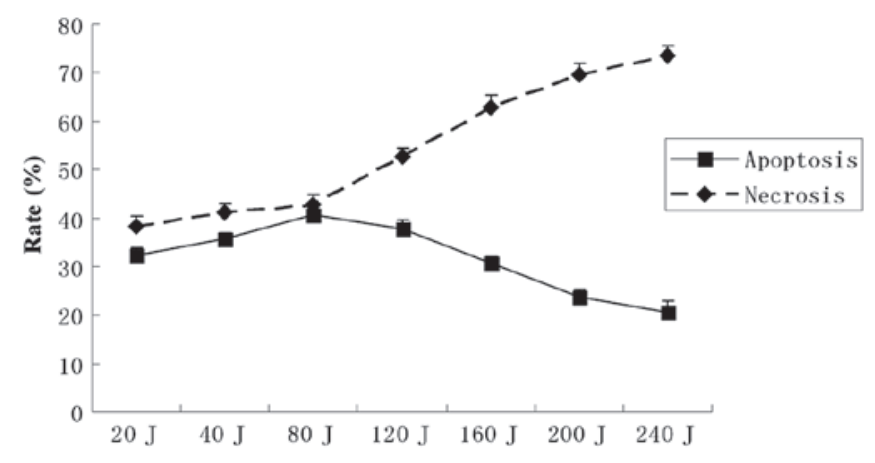

Figure 3. Sonodynamic therapy (SDT) combined with different doses of light irradiation (20-240 J/cm²) induced C6 cell apoptosis or necrosis, evaluated by flow cytometry. Data represent mean $\pm \mathrm{SE}(\mathrm{n}=6)$.

\section{Results}

Inhibition of cell growth with SPDT compared with PDT or SDT. The growth inhibition rate of C6 glioma cells was determined by MTT assay in SDT combined with different

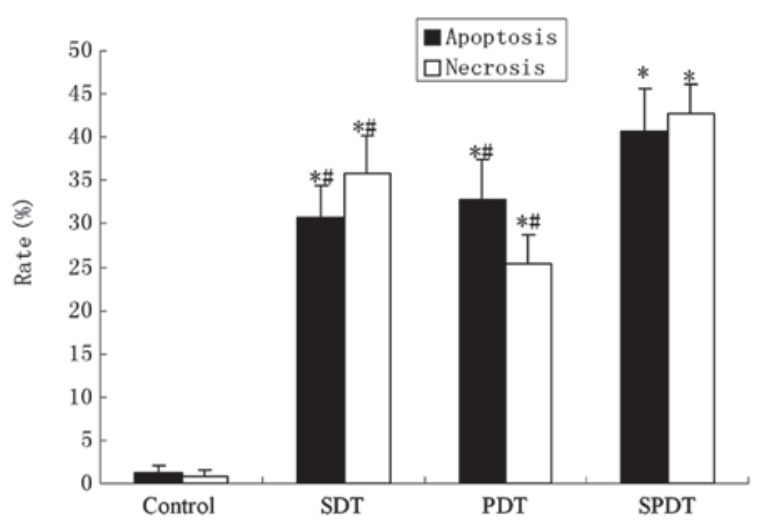

Figure 4. Apoptosis and necrosis measured in rat C6 glioma cells 4 hours after different treatment as measured by flow cytometry of Annexin V-PI treated cells. Data represent mean $\pm \mathrm{SE}(\mathrm{n}=6)$. ${ }^{*} \mathrm{P}<0.05$ vs control;. ${ }^{\text {}} \mathrm{P}<0.05$ vs. SPDT. SDT, sonodynamic therapy; PDT, photodynamic therapy; SPDT, sonodynamic therapy combined with photodynamic therapy.

doses of light irradiation (20-240 J/cm²). Although PDT or SDT alone also inhibited cell growth, the inhibition rate in the SPDT group was significantly higher than that in the SDT or PDT groups at certain doses (light dose $<200 \mathrm{~J} / \mathrm{cm}^{2}$; $\mathrm{P}<0.05)$. When the light dose was $>200 \mathrm{~J} / \mathrm{cm}^{2}$, the inhibition rate was not significantly different from that in the PDT group ( $\mathrm{P}>0.05)$. However, in the SPDT groups, when the light dose was $>120 \mathrm{~J} / \mathrm{cm}^{2}$, the inhibition rate was not significantly increased with increasing light dose $(\mathrm{P}>0.05)$. Thus, our data indicates that HMME-mediated SPDT induced a synergetic killing effect on C6 cells at specific light doses (Figs. 1 and 2). A similar killing effect was achieved by SPDT with lower doses of light compared with PDT alone with higher doses of light.

SDT-induced apoptosis. The flow cytometry assay showed marked changes in the cell profile following SPDT. Using flow cytometry, we evaluated the effect of combining SDT with different doses of light irradiation (20-240 J/ $\mathrm{cm}^{2}$ ) on apoptosis or necrosis in C6 cells. In the SPDT group, the cell apoptosis rate rose with the increasing light dose when the light irradiation dose was $<80 \mathrm{~J} / \mathrm{cm}^{2}$. Conversely, the cell apoptosis rate reduced as the light dose increased when the light dose was $>80 \mathrm{~J} / \mathrm{cm}^{2}$; however, the necrosis rate increased notably when the light dose was $>80 \mathrm{~J} / \mathrm{cm}^{2}$. The results suggested that SPDT induced the highest level of cell apoptosis when SDT was combined with a light irradiation dose of $80 \mathrm{~J} / \mathrm{cm}^{2}$ (Fig. 3). The apoptotic rate was $\sim 40.62 \pm 5.01 \%$ with a light irradiation dose of $80 \mathrm{~J} / \mathrm{cm}^{2}$ in SPDT. The apoptosis rate of C6 glioma cells in the SPDT group was the highest among all groups $(\mathrm{P}<0.05)$, while the SDT and PDT groups were higher compared with the control $(\mathrm{P}<0.01)$, but lower than the SPDT group $(\mathrm{P}<0.05)$. HMMEmediated SPDT also increased the apoptotic rate of C6 cells in certain conditions (Fig. 4).

SPDT induces caspase 3, 8 and 9 activation. As shown by the western blot analysis of cytosolic extracts, HMME-SPDT induced caspase 3,8 and 9 cleavage with a light irradiation dose of $80 \mathrm{~J} / \mathrm{cm}^{2}$ in SPDT. Band intensity quantitation measure- 


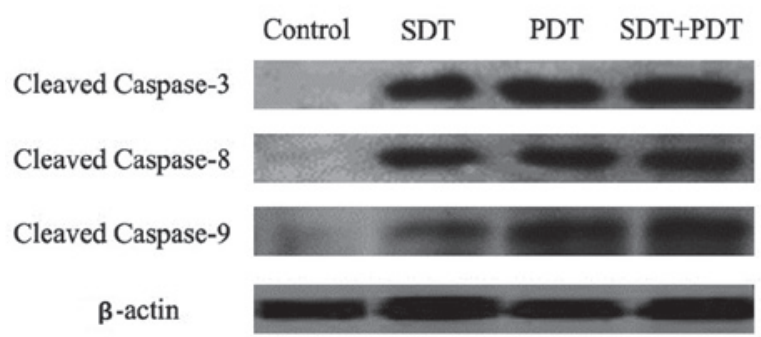

Figure 5. Caspase 3, 8 and 9 cleavage in C6 cells following various treatments. Representative western blots for the expression of caspase 3, 8 and 9 cleavage in different groups. SDT, sonodynamic therapy; PDT, photodynamic therapy; SPDT, sonodynamic therapy combined with photodynamic therapy.

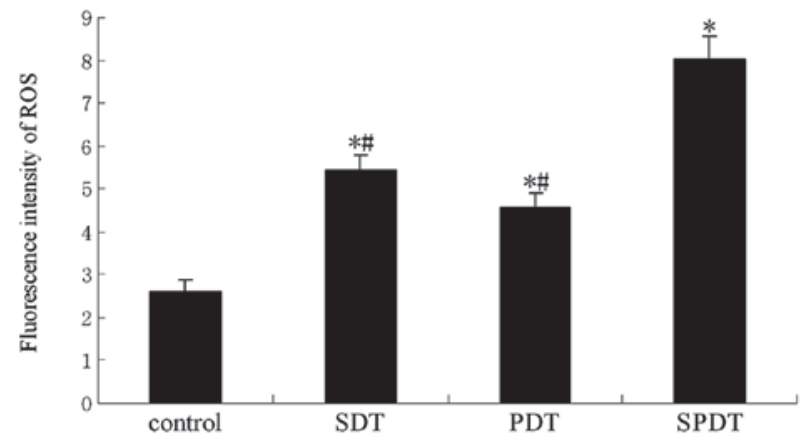

Figure 6. Reactive oxygen species (ROS) production as indicated by DCFH fluorescence in different groups. Data are mean \pm SE $(n=6)$. ${ }^{*} \mathrm{P}<0.05$ vs. control; ${ }^{*} \mathrm{P}<0.05$ vs. SPDT. SDT, sonodynamic therapy; PDT, photodynamic therapy; SPDT, sonodynamic therapy combined with photodynamic therapy.

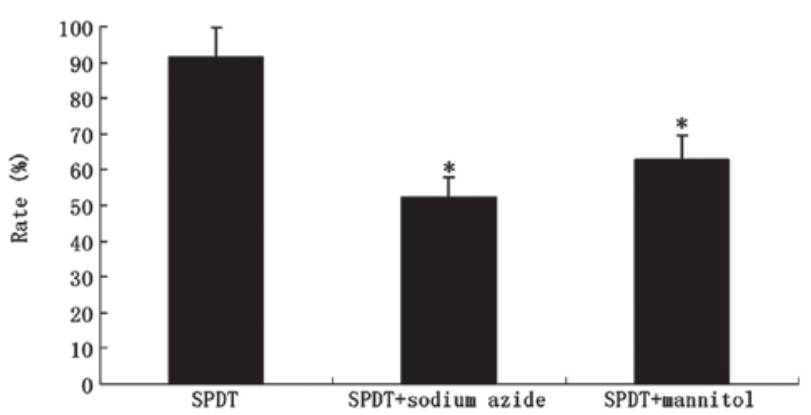

Figure 7. Effect of scavengers on the SPDT-induced cell death (mean \pm SE, $\mathrm{n}=6$ ). Growth inhibition rate of rat C6 glioma cells in different groups were measured by MTT assay. ${ }^{*} \mathrm{P}<0.05$, compared with SPDT group. SPDT, sonodynamic therapy combined with photodynamic therapy.

ments showed that the activation rate of caspase 3,8 and 9 was markedly higher than that in the SDT or PDT groups alone (Fig. 5).

Effect of ROS on SDT-induced cell killing. To demonstrate the effect of ROS in SPDT-induced cell killing, ROS production was confirmed by the oxidation of DCFH-DA. The results showed that the synergistic effect of SDT and PDT generated more ROS than SDT or PDT alone at a light irradiation dose of $80 \mathrm{~J} / \mathrm{cm}^{2}$ in SPDT (P<0.01; Fig. 6). HMME-mediated SPDT killing of glioma cells was studied in the absence or presence of various ROS scavengers (e.g., $\mathrm{NaN}_{3}$ and mannitol). The presence of $\mathrm{NaN}_{3}$ significantly reduced the inhibition rate of HMME-mediated SPDT. At the level of $100 \mu \mathrm{g} / \mathrm{ml}$, the singlet oxygen scavenger caused a reduction of almost $39 \%(\mathrm{P}<0.01)$. The presence of mannitol also reduced HMME-mediated SPDT killing by $29 \%$ ( $\mathrm{P}<0.05$; Fig. 7 ).

\section{Discussion}

SPDT is a new, combined therapy for treating cancer. The basis of the therapy is to administer a small amount of sensitizer, which is selectively taken up by cancer cells, and then expose the body to light and sound to activate these sensitizers (17-19). These techniques are known to have a cancer-killing effect and are applicable to a wide range of cancers, but the effect of killing malignant glioma cells is unknown. We therefore used light and sound to activate the photosensitizer, a hematoporphyrin derivative (HMME), and examined its effectiveness and mechanisms of killing glioma cells.

For evaluating the effects of HMME-mediated SPDT, we first determined the growth inhibition rate of C6 glioma cells in the SPDT group compared with other groups. Our results indicated that HMME-mediated SPDT induced a synergetic killing effect on C6 cells when the light dose was $<200 \mathrm{~J} / \mathrm{cm}^{2}$ (Figs. 1 and 2). SPDT at a lower light dose achieved a better synergetic killing effect than the monotherapies. In the SPDT groups, when the light dose was greater than $120 \mathrm{~J} / \mathrm{cm}^{2}$, the inhibition rate was not significantly increased with increasing light dose $(\mathrm{P}>0.05)$. This indicated that SPDT had a sufficient killing effect when ultrasound generated $1 \mathrm{MHz}$ frequency, $0.5 \mathrm{~W} / \mathrm{cm}^{2}$ intensity and light irradiation up to $120 \mathrm{~J} / \mathrm{cm}^{2}$. SPDT effectively killed C6 cells with lower doses of light compared with PDT alone. Hence, we determined that HMME-mediated SPDT was a good method for killing C6 gliomas.

Apoptosis is one of major modes of tumor cell death (20). In this experiment, we used light and sound to activate the photosensitizer HMME and examined its effectiveness and the mechanisms by which it induces $\mathrm{C} 6$ glioma cell apoptosis. Cell apoptosis was observed in HMME-mediated SPDT in vitro. In our experiment, we revealed that the apoptosis rate of C6 cells significantly increased when the light irradiation dose was lower than $80 \mathrm{~J} / \mathrm{cm}^{2}$; however, when the light irradiation dose was above $80 \mathrm{~J} / \mathrm{cm}^{2}$, the necrosis rate of C6 cells significantly increased instead of apoptosis. Experimental results indicated that HMME-mediated SPDT induced the highest rate of C6 glioma cell apoptosis at a light dose of $80 \mathrm{~J} / \mathrm{cm}^{2}$. These results indicated that SDT and PDT had a synergic effect on inducing tumor cell apoptosis at a certain light dose. It suggests that SPDT with low doses of light may induce major C6 cell apoptosis; but in contrast it mainly results necrosis with high doses of light.

As we know, apoptosis is often initiated by either an extrinsic (activated caspase 8) or an intrinsic pathway (activated caspase 9). The extrinsic pathway functions can directly activate caspase 8 through the death receptors on the cell surface; however, the intrinsic pathway regulates the activation of caspase 9, and subsequently the activation of caspase 3 . In the present study, we attempted to indentify the intrinsic or extrinsic apoptosis pathways using an SPDT in vitro model. 
Our experiment also demonstrated that HMME-mediated SPDT magnified the release of cleaved caspase 3, 8 and 9 in cytoplasm. The changes in the SDT or PDT group were not as apparent as those in the SPDT group. These results showed that both the mitochondrial and death receptor pathway may be two channels by which SPDT induces C6 glioma cell apoptosis.

Certain studies have demonstrated that ROS were generated following by SDT or PDT $(11,21)$. Oxidative stress has been reported to initiate the killing effect in SDT or PDT (22-24). In our experiment, we also detected that SPDT increased ROS levels in vitro (Fig. 6). Although SDT or PDT alone increased the generation of ROS, ROS generation in these groups was much lower than that in the SPDT group. The presence of $\mathrm{NaN}_{3}$ or mannitol significantly reduced the inhibition rate of HMME-mediated SPDT. It suggested that increased ROS generation was the key factor to killing C6 cells. These results are similar to those found in other reports $(12,13)$.

In summary, these results suggest that HMME-mediated SPDT may be a promising method for the treatment of glioma. Combined SDT with PDT at various conditions may cause different biological effects.

\section{Acknowledgements}

This study was supported by the National Natural Science Foundation (81072079), the Technological Key Research Projects of Heilongjiang Province (GC10C304-1), and Bureau of Health Foundation of Heilongjiang Province, China Province (2010-129).

\section{References}

1. Calzavara-Pinton PG, Venturini M and Sala R: Photochemistry and photobiology. J Eur Acad Dermatol Venereol 21: 293-302, 2007.

2. Kessel D, Jeffers R, Fowlkes JB and Cain C: Effects of sonodynamic and photodynamic treatment on cellular thiol levels. J Photochem Photobiol B 32: 103-106, 1996.

3. Worthington AE, Thompson J, Rauth AM and Hunt JW: Mechanism of ultrasound enhanced porphyrin cytotoxicity. Part I: a search for free radical effects. Ultrasound Med Biol 23 : 1095-1105, 1997.

4. Yumita N and Umemura S: Sonodynamic therapy with photofrin II on AH130 solid tumor. Pharmacokinetics, tissue distribution and sonodynamic antitumoral efficacy of photofrin II. Cancer Chemother Pharmacol 51: 174-178, 2003.

5. Rosenthal I, Sostaric JZ and Riesz P: Sonodynamic therapy - a review of the synergistic effects of drugs and ultrasound. Ultrason Sonochem 11: 349-363, 2004.

6. Yumita N, Nishigaki R, Umemura $\mathrm{K}$ and Umemura $\mathrm{S}$ : Hematoporphyrin as a sensitizer of cell-damaging effect of ultrasound. Jpn J Cancer Res 80: 219-222, 1989.
7. Kessel D, Jeffers R, Fowlkes JB and Cain C: Porphyrin-induced enhancement of ultrasound cytotoxicity. Int J Rad Biol 66: 221, 1994.

8. Miyoshi N, Misik V and Riesz P: Sonodynamic toxicity of gallium-porphyrin analogue ATX-70 in human leukemia cell. Radiat Res 148: 43-47, 1997.

9. Umemura S, Yumita N, Umemura $K$ and Nishigaki R: Sonodynamically induced effect of rose bengal on isolated sarcoma 180 cells. Cancer Chemother Pharmacol 43: 389-393, 1999.

10. YumitaN,KawabataK,SasakiKandUmemuraS:Sonodynamic effect of erythrosin B on sarcoma 180 cells in vitro. Ultrason Sonochem 9: 259-265, 2002.

11. Tachibana K, Uchida T, Ogawa K, Yamashita N and Tamura K: Induction of cell-membrane porosity by ultrasound. Lancet 353 : 1409, 1999.

12. Kessel D, Lo J, Jeffers R, Fowlkes JB and Cain C: Modes of photodynamic vs. sonodynamic cytotoxicity. J Photochem Photobiol B 28: 219-221, 1995

13. Jin ZH, Miyoshi N and Ishiguro K: Combination effect of photodynamic and sonodynamic therapy on experimental skin squamous cell carcinoma in $\mathrm{C} 3 \mathrm{H} / \mathrm{HeN}$ mice. Dermatol 27: 294-306, 2000.

14. Kolarova H, Tomankova K, Bajgar R, Kolar P and Kubinek R: Photodynamic and sonodynamic treatment by phthalocyanine on cancer cell lines. Ultrasound Med Biol 35: 1397-1404, 2009.

15. Dunn F, Averbuch AJ and O'Brien WD Jr: A primary method for the determination of ultrasonic intensity with the elastic sphere radiometer. Acoustica 38: 58-61, 1977.

16. Li JH, Song DY, Xu YG, Zheng Huang and Yue Wu: In vitro study of haematoporphyrin monomethyl ether-mediated sonodynamic effects on C6 glioma cells. Neurol Sci 29: 229-235, 2008.

17. Liu QH, Wang P, Li M, et al: Apopotosis of Ehrlich ascites tumor cells by sonochemical activated hematoporphyrin. Acta Zool Sinica 49: 620, 2003.

18. Tang W, Liu Q, Wang X, et al: Involvement of caspase 8 in apoptosis induced by ultrasound-activated hematoporphyrin in sarcoma 180 cells in vitro. J Ultrasound Med 27: 645-656, 2008.

19. Wang XB, Liu QH, Wang P, et al: Enhancement of apoptosis by sonodynamic therapy with protoporphyrin IX in isolate sarcoma 180 cells. Cancer Biother Radiopharm 23: 238-246, 2008.

20. Rieger L, Weller M, Bornemann M, et al: BCL-2 family protein expression in human malignant glioma: a clinical-pathological correlative study. J Neurol Sci 155: 68-75, 1998.

21. Yumita N, Nishigaki R, Umemura K, et al: Sonochemical activation of hematoporphyrin: an ESR study. Radiat Res 138: 171-176, 1994.

22. Rogalska A, Koceva-Chyła A and Jóźwiak Z: Aclarubicininduced ROS generation and collapse of mitochondrial membrane potential in human cancer cell lines. Chem Biol Interact 176: 58-70, 2008.

23. Das R, Roy A, Dutta N and Majumder HK: Reactive oxygen species and imbalance of calcium homeostasis contributes to curcumin induced programmed cell death in Leishmania donovani. Apoptosis 13: 867-882, 2008.

24. Yang J, Wu LJ, Tashino S, Onodera S and Ikejima T: Critical roles of reactive oxygen species in mitochondrial permeability transition in mediating evodiamine-induced human melanoma A375-S2 cell apoptosis. Free Radic Res 41: 99-108, 2007. 